\title{
Rapid Method Using Deep Learning with Multi-focus Microphotographs to Measure Submicrometric Structures and Its Evaluation
}

\author{
Riki Narukage, George Okada, and Hiroshi Kawaguchi* \\ Graduate School of Science, Technology and Innovation, Kobe University, Japan \\ ${ }^{*}$ Corresponding author's e-mail: kawapy@godzilla.kobe-u.ac.jp
}

\begin{abstract}
Confocal laser scanning microscopy (CLSM) and scanning electron microscope (SEM) systems are commonly used for measuring the dimensions of laser-processed objects. Nevertheless, both methods require some time for preprocessing and measurement, thereby entailing high costs. We propose a simple, fast, and inexpensive method for measuring submicrometric structures using deep learning with multi-focus microphotographs taken using an optical microscope. The average errors in depth and height for a laser-processed groove and a laser-processed ridge are, respectively, 0.1667 $\mu \mathrm{m}$ and $0.4349 \mu \mathrm{m}$. The estimation time is $971.50 \mathrm{~ms}$ for the $64 \times 64 \mu \mathrm{m}^{2}$ area.
\end{abstract}

DOI: $10.2961 /$ jlmn.2021.02.3001

Keywords: machine learning, microphotograph, submicrometric structure measurement

\section{Background}

Semiconductor manufacturing has increasingly demanded precise processing [1]. Particularly for semiconductor packaging or so-called "postprocessing”, an interposer, which mounts a die cutout from a silicon wafer, requires submicrometer-level processing dimensions. Similarly, on the surface of a ceramic electrostatic chuck for fixing a silicon wafer, processing in submicrometer order is necessary [2]. In these applications, laser processing can achieve high yields. Nevertheless, many difficulties hinder measurement of the process results.

Confocal laser scanning microscopy (CLSM), SEM systems, and probes [3] are generally used for measuring dimensions. These devices can measure dimensions precisely, but they are expensive and require long measuring times. For those reasons, they cannot take full advantage of fast laser processing. For example, a laser microscope takes about 40 s to measure an area of $128 \times 128 \mu \mathrm{m}^{2}$. By optical microscopy, some studies have realized the measurement of sub$100 \mathrm{~nm}$ feature size, combining optical science and informatics [4] [5]. However, these works do not mention the cost or measurement time. Therefore, it may not be possible to implement them directly in manufacturing sites. In these respects, this paper is practical. In semiconductor industry, which is the subject of this article, cost and speed efficiency are sometimes more crucial than resolution [6].

Therefore, this study aims to develop a new method in line with the following concepts.

- Low-cost systems only with an optical microscope

- High-speed machine learning for shape estimation

- A reproducible evaluation method that does not rely on the human eye

In the manufacturing process, accuracy is improved through evaluation as well as measurement of the machining results. In this research, we aim to develop a system that automatically measures and evaluates the processing results, rather than just a measuring device.

Our system uses multi-focus microphotographs taken by a low-cost optical microscope to find cross-section information related to a substrate. The system then creates virtual cross-sectional images using high-speed machine learning. The processing flow is presented in Fig. 1.

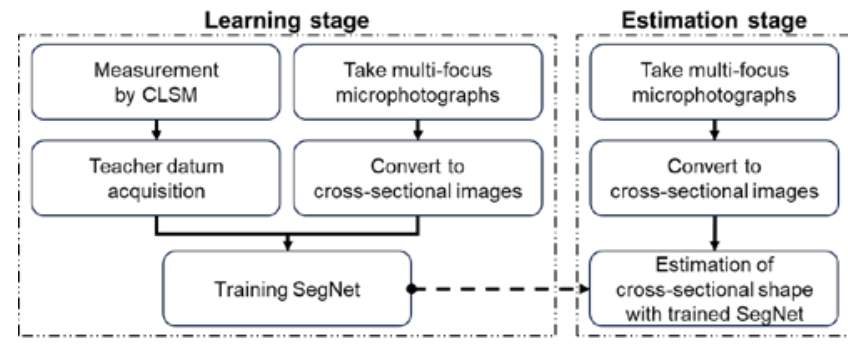

Fig. 1 Process flow.

\section{Semantic Segmentation}

We use a deep learning algorithm called "semantic segmentation” to label each pixel in an image. By this technique, a cross-sectional shape is estimated from a virtual cross-sectional image. This approach resembles the original semantic segmentation mission, which distinguishes all objects in an image.

Several neural networks are used for semantic segmentation. We take SegNet [7] to estimate the cross-sectional shape. Compared to other neural networks, SegNet features a special "convolutional encoder-decoder structure" in the figure, which makes it possible to label objects of various sizes in the image through the network. U-Net [8], which is more accurate than SegNet, is known as a network for semantic segmentation. However, because the microscopic images used in this study are very noisy, the accuracy of UNet is almost the same as that of SegNet. On the other hand, SegNet was more advantageous in terms of execution time; U-Net takes about twelvefold time for training and about double time for estimation. Therefore, we adopted SegNet in this study.

\section{Implementation}

For experiments, an alumina ceramic substrate, which is also used for the electrostatic chuck device, is used. It is durable, white-colored, and has little reflectivity: transparent 
materials are unsuitable for micrographs. Processes of two types are prepared to produce a laser-processed groove (concave) and a laser-processed ridge (trapezoidal convex).

\subsection{Laser-processed groove}

Laser processing is performed on the alumina ceramic material to produce seven grooves, for which processing parameters are varied. These grooves have depths of 8-10 $\mu \mathrm{m}$.

\subsection{Laser-processed ridge}

A single ridge of $8 \mu \mathrm{m}$ height is a sample made by shaving the alumina ceramic periphery with a laser.

\subsection{Virtual cross-sectional images}

Multi-focus microphotographs are obtained by changing only the focal planes in the vertical direction while a view range is fixed for the photographic conditions. Fig. 2 shows a cross-sectional image of the material converted from the multi-focus microphotographs. The image range is $257 \times$ $128 \mu \mathrm{m}^{2}$ for each. The focal planes are at a pitch of $0.5 \mu \mathrm{m}$ in the depth direction. For the same image range, 40 depth images are taken. Appendix A presents pseudo-code for taking cross-sectional images.

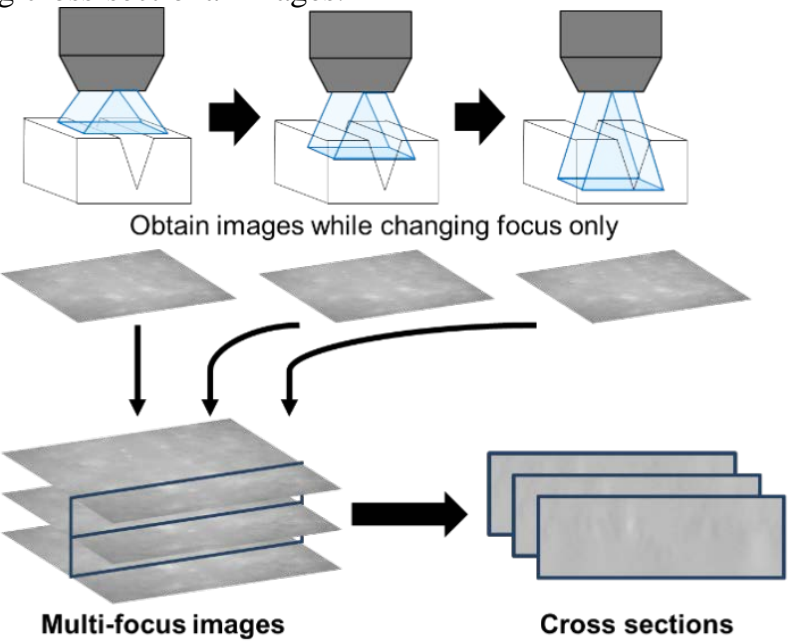

Fig. 2 Cross-section images converted from multi-focus images (optical microscope OLS4100, Olympus Corp.).

\subsection{Teacher data, training data, and validation data}

Teacher data for the cross-sectional images are obtained using a laser microscope for the same image range. Fig. 3 is a sample pair of a cross-sectional image and a teacher image. The image size of a teacher image is $512 \times 32$ pixels. Regarding training data and validation data, augmented images of $128 \times 32$ pixels are cut out randomly from the original cross-section images, as shown in Fig. 4.

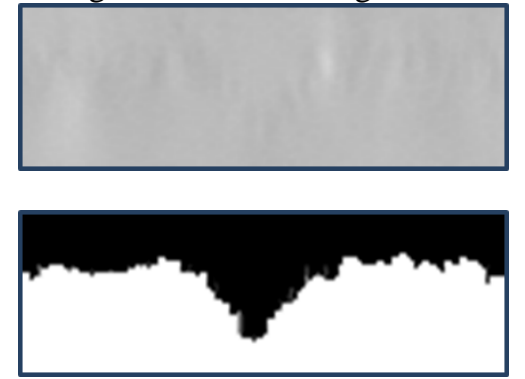

Fig. 3 Pair of a cross-section image and teacher data.

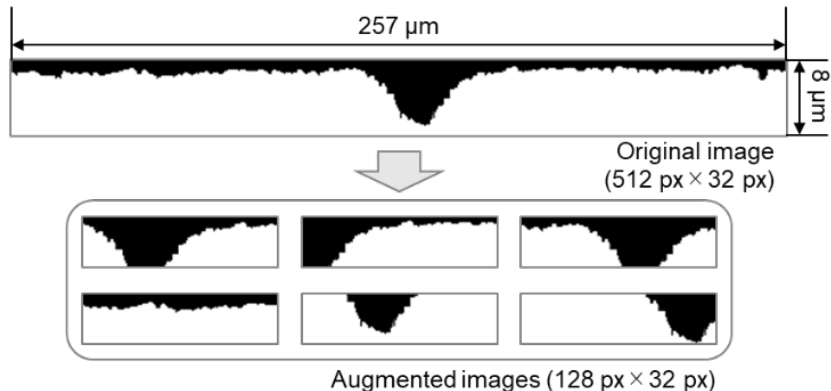

Fig. 4 Augmented images for training data and validation data cut out from an original cross-section image.

\section{Estimation Results with SegNet Four Layers}

\subsection{Laser-processed groove}

It took 3,900 s to train 64,744 images on a PC (Core i78700@3.20 GHz; Intel Corp. and GeForce GTX 1080; Nvidia Corp.). By inference, the shape estimation was completed in $21.39 \mathrm{~s}$ for 6,144 validation data, resulting in an accuracy of 0.9543 on pixel bases (Table I). Fig. 5 presents a sample pair of validation data: a virtual cross-section image and an estimated cross-section, which is located near the position of Fig. 3; they exhibit the same characteristics. The estimated groove is expanded to a whole shape. Fig. 6(a) portrays a measured groove structure with a laser microscope, whereas Fig. 6(b) is an estimated one with SegNet; each comprises 256 cross-sections. The area is $64 \times 64 \mu \mathrm{m}^{2}$. The estimation time is $971.50 \mathrm{~ms}$.

The laser light output decreases radially from the center according to a normal distribution. Grooving is one-stroke processing. Therefore, it can be assumed that the shape after processing is close to a normal distribution graph. We tried to fit the grooving shape to the normal distribution graph for approximation. Three parameters: groove width $w$, groove center $c$, and groove depth $d$ were introduced as normal distribution graph parameters. Then, we compared the three differences parameters between the measured shape with a laser microscope and the estimated shape with SegNet. The average difference was calculated from the values of the laser microscope. The average $\mu$ and standard deviation $\sigma$ of the differences between the measured and estimated ones exhibit accuracies of the estimated groove shapes. Fig. 7 shows fitting examples and accuracies of the estimated shapes when the fitting curves are, respectively, one-sigma, twosigma, and three-sigma normal distribution graphs. Appendix. B is a pseudo-code of the detailed algorithm for fitting to the normal distribution graph.

Table I Accuracy for a laser-processed groove on pixel ba-

\begin{tabular}{|c|c|c|c|}
\hline \multicolumn{2}{|c|}{} & \multicolumn{2}{c|}{ Actual } \\
\cline { 2 - 4 } \multicolumn{2}{|c|}{} & Positive & Negative \\
\hline \multirow{3}{*}{ Prediction } & Positive & $\mathbf{0 . 9 3 7}$ & 0.063 \\
\cline { 2 - 4 } & Negative & 0.037 & $\mathbf{0 . 9 6 3}$ \\
\hline
\end{tabular}



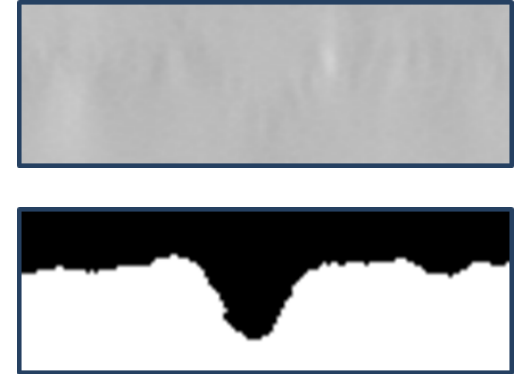

Fig. 5 Pair of a cross-sectional image and validation data.

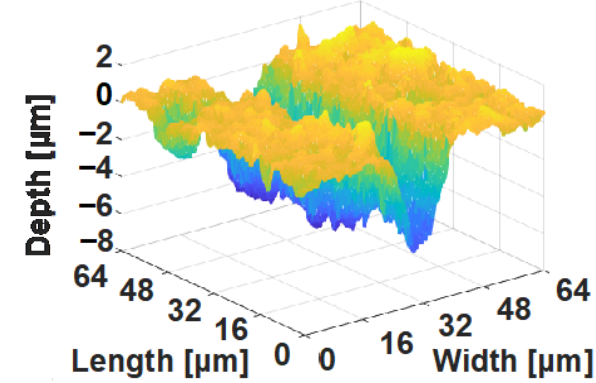

(a)

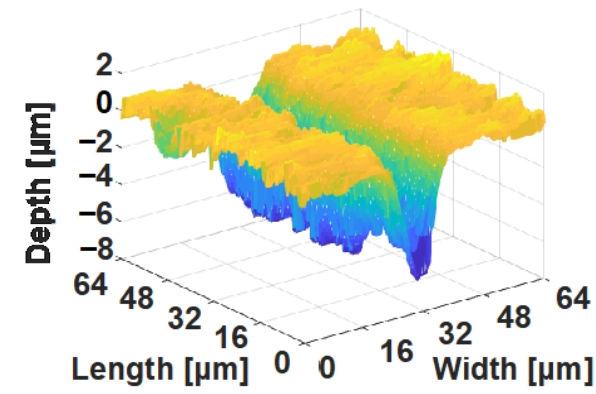

(b)

Fig. 6 (a) Measured groove structure with a laser microscope (OLS4100, Olympus Corp.) and (b) estimated one with SegNet.

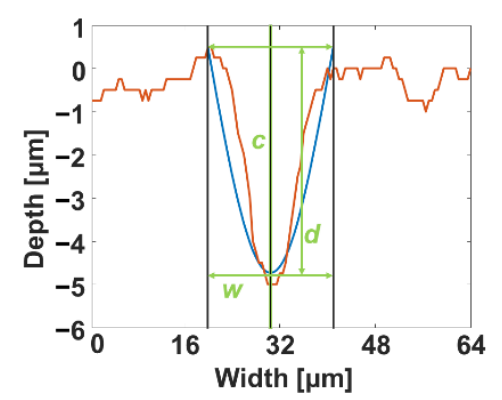

\begin{tabular}{|c|c|c|}
\hline & $\boldsymbol{\mu}$ & $\boldsymbol{\sigma}$ \\
\hline width: $\boldsymbol{w}[\mu \mathrm{m}]$ & $-\mathbf{1 . 6 4 0 6}$ & $\mathbf{2 . 8 9 1 0}$ \\
\hline center: $\boldsymbol{c}[\mu \mathrm{m}]$ & $-\mathbf{0 . 3 9 8 4}$ & 0.6997 \\
\hline depth: $\boldsymbol{d}[\mu \mathrm{m}]$ & 0.3426 & 1.7339 \\
\hline
\end{tabular}

(a)

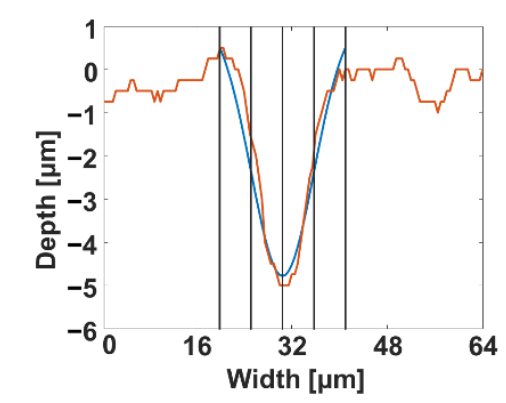

\begin{tabular}{|c|c|c|}
\hline & $\boldsymbol{\mu}$ & $\boldsymbol{\sigma}$ \\
\hline width: $\boldsymbol{w}[\mu \mathrm{m}]$ & -1.9609 & 2.9226 \\
\hline center: $\boldsymbol{c}[\mu \mathrm{m}]$ & -0.4102 & $\mathbf{0 . 6 5 9 4}$ \\
\hline depth: $\boldsymbol{d}[\mu \mathrm{m}]$ & $\mathbf{0 . 1 6 7 6}$ & 0.8146 \\
\hline
\end{tabular}

(b)

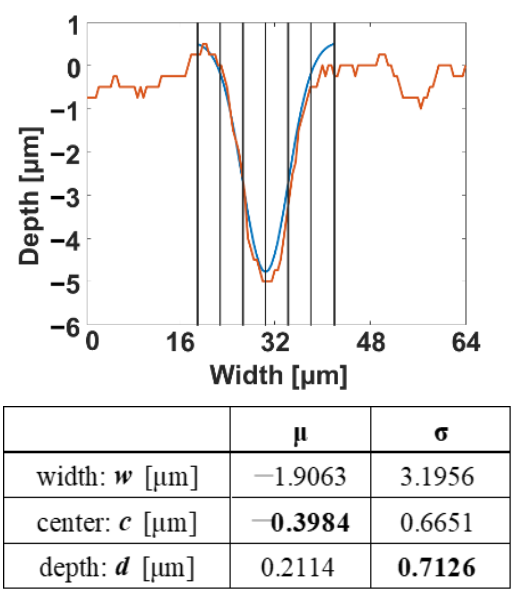

(c)

Fig. 7 Fitting examples and accuracies of the groove structure for normal distribution approximations: (a) one sigma,

(b) two sigma, and (c) three sigma.

\subsection{Laser-processed ridge}

We prepared 61,440 training data and 10,240 validation data for the single ridge. Table II shows that the accuracy is 0.9148 on pixel bases. Figs. 8(a) and 8(b) respectively depict a ridge structure measured with a laser microscope and a ridge structure estimated using SegNet. For graph fitting, height $h$, upper base $u$, lower base $l$, and center c were defined as parameters because a cross-section of a ridge is regarded as a trapezoid. Fig. 9 shows a fitting example and accuracies of the estimated shapes. Appendix. C presents pseudo-code of the algorithm for fitting to the trapezoid.

Table II Accuracy in laser-processed ridge on pixel bases

\begin{tabular}{|c|c|c|c|}
\hline \multicolumn{2}{|c|}{} & \multicolumn{2}{c|}{ Actual } \\
\cline { 3 - 4 } & Positive & Negative \\
\hline \multirow{3}{*}{ Prediction } & Positive & $\mathbf{0 . 9 2 2}$ & 0.078 \\
\cline { 2 - 4 } & Negative & 0.096 & $\mathbf{0 . 9 0 4}$ \\
\hline
\end{tabular}




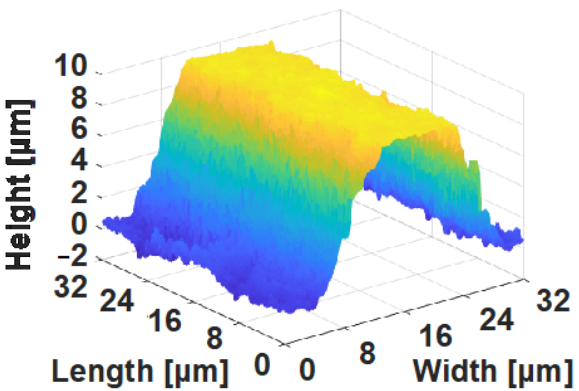

(a)

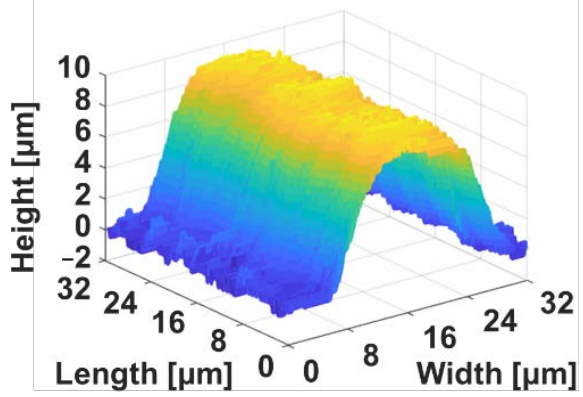

(b)

Fig. 8 (a) Ridge structure measured with a laser microscope (OLS4100, Olympus Corp.) and (b) estimated using SegNet.

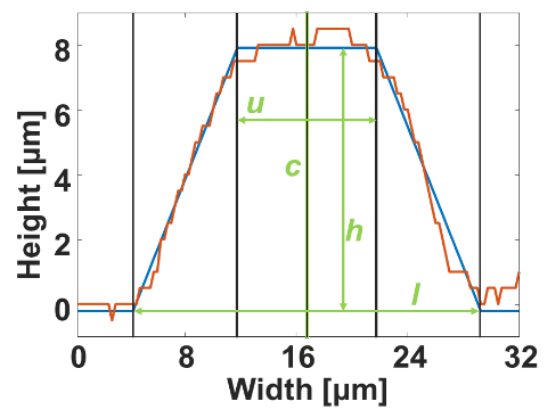

\begin{tabular}{|c|c|c|}
\hline & $\boldsymbol{\mu}$ & $\boldsymbol{\sigma}$ \\
\hline height: $\boldsymbol{h}[\mu \mathrm{m}]$ & 0.4349 & 0.2806 \\
\hline upper base: $\boldsymbol{u}[\mu \mathrm{m}]$ & 0.0859 & 1.3560 \\
\hline lower base: $\boldsymbol{l}[\mu \mathrm{m}]$ & 0.9570 & 1.4410 \\
\hline center: $\boldsymbol{c}[\mu \mathrm{m}]$ & -0.0869 & 0.4183 \\
\hline
\end{tabular}

Fig. 9 Fitting example and accuracies of the ridge structure for trapezoid approximation.

\section{Summary and Discussion}

Our proposed method estimated dimensions in laser-processed submicrometric structures. By fitting the laser-processed groove with the proposed evaluation method, the average errors of ridge depth and height are $0.1667 \mu \mathrm{m}$ and $0.4349 \mu \mathrm{m}$, respectively, in comparison with the proposed method and the conventional shape measuring instrument. The accuracy reached submicrometer order. High-speed shape estimation is possible by combining a computer and a microscope.

Since the proposed method requires pre-training, it is suitable for mass production, for example, defect detection on a manufacturing line. Further experiments with various imaging conditions and materials are needed to determine whether our proposed method can be used for other purposes.

In this paper, the experiments were performed only on the white, durable, and low-reflective ceramic since we focused on ceramic interposers and ceramic electrostatic chucks used in semiconductor manufacturing. The proposed method, however, processes a monochrome image in machine learning; color is not an issue. We believe that it is possible to measure less durable material that are easily cracked or debris-prone. This is because the shape can be estimated as far as its surface can be viewed with an optical microscope. On the other hand, it would be difficult to use this method for reflective materials because the surface cannot be observed with an optical microscope.

The proposed method may be used not only for laser grooving but also for laser ablation processing. Observing micro-surface is important [9] [10]. Our proposed method is applicable to observing operations with submicrometric accuracy, where pre-learning is possible and real-time shape estimation in repeated processing is desirable.

\section{References}

[1] "Opportunities for the global semiconductor market," https://www.pwc.com/gx/en/industries/tmt/publications/global-tmt-semiconductor-report-2019.html.

[2] Y. Moriya, K Ando: U. S. Patent 10389278B2 (2016).

[3] M. C. Fischer, J. W. Wilson, F. E. Robles, and W. S. Warren: Rev. Sci. Instrum. 87, (2016) 031101.

[4] L. Chen, Y. Zhou, Y. Li, and M. Hong: Appl. Phys. Rev. 6, (2019) 021304.

[5] L. Chen, Y. Yin, Y. Li, and M. Hong: Opto-Electron. Adv. 2, (2019) 190019.

[6] L. Chen, Y. Zhou, Z. Rui, and M. Hong: iScience, 23, (2020) 101211.

[7] V. Badrinarayanan, A. Kendall, and R. Cipolla: IEEE Trans. Pattern Anal. Mach. Intell., 39, (2017) 2481

[8] O. Ronneberger, P. Fischer, and T. Brox: Proc. MICCAI 2015, (2015) 234

[9] Livakas N, Skoulas E, Stratakis E: Opto-Electron. Adv. 3, (2020) 190035

[10] Papanikolaou A, Tserevelakis G J, Melessanaki K, Fotakis C, Zacharakis G et al.: Opto-Electron. Adv. 3, (2020) 190037

\section{Appendix}

Appendix A Pseudo code for taking cross-section images. declare the array[height][width][num of images]

for $k=1$ to num of images

open images into the array $[:][:][\mathrm{k}]$

for $i=1$ to height export images from the array[i][:][:]

Appendix B Pseudo code for fitting to a normal distribution for Wavelength $=1$ to length of $\backslash$ the cross_sectional_wave make normal distribution wave with \ the same length as Wavelength

calculate convolution between \ cross_sectional_wave and \ normal distribution wave search maximum value in calculation result

substitute the maximum value into the array 
search maximum value in the array and $\backslash$ get the value of center " $c$ " and width " $w$ "

make the distribution_wave from " $c$ " and " $w$ " calculate the cross_sectional_wave * ।

the distribution_wave / sum of squared elements \ of the distribution_wave

get the stretched_distribution_wave $\backslash$

from the distribution_wave multiplied \

by the culculation result

get the value of depth " $d$ " from differences \ between maximum value and minimum value $\backslash$

in the stretched distribution wave

Appendix C Pseudo code for fitting to a trapezoid.

for lower_base $=3$ to length $\backslash$

of the cross_sectional_wave

for topper_base $=$ lower base- 2 to 1

declare height $=1$

make normal_trapezoid_wave ।

from lower_base and topper_base

calculate cross-correlation

between cross_sectional_wave I

and normal_trapezoid_wave

substitute calculate result ।

into the array

search maximum value in the array $\backslash$

and get the value of upper base " $u ", \backslash$

lower_base " $l$ " and center " $c$ "

get the value of height " $h "$ ।

from maximum value in the array

(Received: February 2, 2021, Accepted: April 9, 2021) 